\title{
El papel de la reputación del Consejero Delegado en la reputación de la empresa española*
}

\author{
Urra Urbieta, José Anastasio** \\ Mohedano Suanes, Antonia*** \\ Safón Cano, Vicente ${ }^{* * *}$
}

\section{Resumen}

La reputación corporativa se conceptúa como la estimación global de las percepciones que tienen de la empresa sus principales stakeholders. La literatura ha visto en la reputación corporativa uno de los activos intangibles más valioso desde el punto de vista de la creación y sostenimiento de ventajas competitivas, por lo que diversos estudios han tratado de identificiar los antecedentes de la reputación de la empresa. Uno de estos antecedentes destacado son sus directivos, y entre ellos los Consejeros Delegados. En España, los trabajos sobre los antecedentes de la reputación corporativa son muy escasos, e inexistentes los que han abordado el estudio de la influencia de la reputación del Consejero Delegado sobre la reputación corporativa, hecho que justifica nuestro estudio y se plantea como su objetivo. A partir de una muestra obtenida del Monitor Español de Reputación Corporativa, tras proponer varias hipótesis derivadas de la literatura y de un primer análisis univariante de los datos, hemos realizado un análisis multivariable de regresión múltiple. Nuestros resultados ponen de manifiesto que, para el caso español, las variables grado de concentración sectorial, crecimiento de las ventas y reputación del Consejero Delegado se manifiestan como antecedentes y predictores de la reputación corporativa, lo que permite contemplar y analizar la reputación corporativa desde una perspectiva más operativa.

Palabras clave: Reputación corporativa, reputación del Consejero Delegado, España, análisis multivariable.

Recibido: 02-06-09. Aceptado: 20-10-09

* Este trabajo se enmarca dentro del proyecto de investigación UV-AE-08-2290 de la Universidad de Valencia, España.

** Doctor en Ciencias Económicas y Empresariales, Profesor Titular, Universidad de Valencia (España), E-mail: Anastasio.Urra@uv.es

*** Doctora en Ciencias Económicas y Empresariales, Profesor Ayudante, Universidad de Valencia (España), E-mail: Antonia.Mohedano@uv.es

**** Doctor en Ciencias Económicas y Empresariales, Profesor Titular, Universidad de Valencia (España), E-mail: Vicente.Safon@uv.es 


\title{
The Role of CEO Reputation in the Reputation of the Spanish Firm
}

\begin{abstract}
Corporate reputation can be seen as a global estimate of perceptions held by the firm's major stakeholders. Academics have seen corporate reputation as one of the most valuable intangible assets from the viewpoint of creating and sustaining competitive advantage; therefore, research has tried to identify corporate reputation antecedents. One of these outstanding corporate reputation antecedents is the reputation of the Chief Executive Officer. In Spain, research on corporate reputation antecedents is very scarce, particularly research on the influence of Chief Executive Officer reputation on corporate reputation, a fact that justifies this study and proposes its objective. Based on a sample obtained from the Spanish Corporate Reputation Monitor, and after proposing various hypotheses derived from literature and from preliminary, univariable data analysis, a multivariable regression analysis was made. Results show that, in the case of Spain, sectorial concentration level, sales growth and CEO reputation are the antecedents and best predictors of corporate reputation. These results make it possible to look at and analyze corporate reputation from a more operative perspective.
\end{abstract}

Key words: Corporate Reputation, CEO reputation, Spanish company, multivariable analysis.

\section{Introducción}

El concepto de reputación de una empresa es atributivo por naturaleza, ya que la reputación de una empresa se forma a partir de los procesos de inferencia que sobre sus acciones pasadas y presentes establecen otros agentes con los que se relaciona de forma directa o indirecta (Weigelt \& Camerer, 1988; Kranton, 1996; Thompson, 1996) ${ }^{1}$. La reputación de cualquier empresa depende, por tanto, de otras organizaciones y agentes que realizan juicios sobre la actuación pasada de aquélla, utilizándolos como base para la formación de sus creencias sobre el comportamiento futuro de dicha empresa (Weigelt \& Camerer, 1988; Thompson, 1996). Así, la reputación de la empresa puede conceptuarse como la estimación global de las percepciones que de ella tienen sus principales grupos de interés, o stakeholders, fruto de las experiencias directas e indirectas que los individuos mantienen con la empresa, en un marco de comparación entre rivales (Chun, 2005; Gotsi \& Wilson, 2001).

Adicionalmente, aunque en línea paralela, la literatura ha visto en la reputa-

1 En general, la inferencia es un proceso en el que se va más allá de la información disponible, pretendiéndose llegar a unas conclusiones acerca de unos datos que no están completamente contenidas en los datos mismos; ateniéndose exclusivamente a los datos disponibles no hay inferencia. Los procesos de atribución son un tipo particular de procesos de inferencia; se trata de inferencias causales, o aquellas cuyo propósito es establecer la razón o causa de un hecho observado (Nisbett \& Ross, 1980, por ejemplo, ofrecen una profunda discusión de estas ideas). 
Papel de la reputación del consejero delegado en la reputación de la empresa española Urra Urbieta, José Anastasio; Mohedano Suanes, Antonia y Safón Cano, Vicente

ción uno de los activos intangibles más valioso desde el punto de vista de la creación y sostenimiento de ventajas competitivas (Hall, 1992), y más difíciles de imitar (Dhalla \& Carayannopoulos, 2006). Las empresas con una buena reputación atraen a los mejores empleados y retienen con mayor facilidad a los propios, pueden primar sus precios y necesitar menos esfuerzo comercial que los competidores con menos reputación (coste de la fuerza de ventas, publicidad, entre otros), además de que suelen disfrutar de unos clientes más fieles y satisfechos (Davis, Chun, Vinhas da Silva \& Roper, 2003; Fombrun \& Gardberg, 2000; Fombrun, Gardberg \& Sever, 2000), lo cual se traduce en una ventajas competitivas y una rentabilidad financiera superiores ${ }^{2}$. El informe Corporate Reputation Watch (Harris Interactive, 2002), realizado sobre una muestra de 800 Chief Executie Officers (CEO's) y directivos de USA, Canadá, Bélgica, Italia, Alemania, Reino Unido y Holanda, arroja los siguientes resultados, por este orden, en cuanto a los objetivos empresariales que más se ven favorecidos por la reputación: incremento de las ventas, promoción de transacciones e iniciativas estratégicas, reclutamiento $y$ retención de empleados, y mejora en la cotización de las acciones.

Diversos estudios han tratado de identificiar los antecedentes o dimensiones de la reputación de la empresa. Entre los más recientes, el Reputation Institute
(Fombrun, 2006) ha propuesto siete conjuntos de aspectos como causas fundamentales de la reputación corporativa, a saber, los aspectos relacionados con el producto (calidad, relación calidad-precio, entre otros), con la innovación (rapidez en el traslado de las innovaciones al mercado, rapidez en la adaptación ante el cambio, entre otros), con el clima laboral (retribución a los empleados, oportunidades laborales, entre otros), con el gobierno corporativo (abierto y transparente, ético, entre otros), con la ciudadanía (responsabilidad medioambiental, influencia positiva sobre la sociedad, etc.), con el liderazgo (adecuada organización, posesión de directivos excelentes, etc.), y con el desempeño financiero (rentabilidad, expectativas de crecimiento, etc.). Rindova, Williamson, Petkova \& Sever (2005) han propuesto conceptuar la reputación corporativa como un constructo perceptual basado en dos factores: la calidad percibida y la prominencia o notoriedad de la empresa, siendo la primera, además, causa de la segunda. Según estos investigadores, los antecedentes de la calidad percibida de la empresa son la calidad de los inputs que la empresa utiliza para elaborar el producto y/o prestar el servicio, y la calidad de los activos productivos utilizados por la empresa (por ejemplo, poseer científicos de renombre en el caso de una empresa del sector de la biotecnología). La prominencia, por su parte, encuentra su origen en el papel jugado por los "actores institucionales" (agencias de certifica-

2 Existe una gran cantidad de trabajos que han demostrado la relación entre la reputación y el desempeño financiero; una magnífica compilación de esta literatura y de sus resultados puede verse en De la Fuente Sabaté \& Puente (2003b), entre otros. 
ción, prensa especializada, personajes influyentes, entre otros), quienes evalúan y divulgan información sobre las empresas, formando una especie de opinión pública, que es posteriormente captada por los individuos.

Uno de los antecedentes de la reputación destacado por la literatura son sus directivos, y entre ellos, los Consejeros Delegados (CD). Estos directivos influyen sobre la reputación de la empresa en su rol de activo productivo y a través de su liderazgo, además de por su notoriedad o prominencia en la opinión pública, especialmente entre los inversores y empleados (Helm, 2006). Estudios recientes (Gaines-Ross, 2000) han puesto de manifiesto que la reputación del CD puede llegar a suponer hasta el $50 \%$ de la reputación de la empresa, lo que convierte a esta variable en uno de los antecedentes más importantes de la reputación corporativa. Para la consultora, en Relaciones Públicas y Comunicación, Burson-Marsteller, la razón que explica esta relación causal es que el CD o CEO es, ahora más que nunca, el rostro público y brújula ética de la organización.

En España, son muy escasos los trabajos sobre los antecedentes de la reputación de las empresas españolas (De la Fuente Sabaté \& Puente, 2003a) y, hasta donde conocen los autores, inexistentes los que han abordado el estudio de la influencia de la reputación del CD sobre la reputación corporativa, hecho que justifica nuestro estudio y se plantea como objetivo del artículo.

A continuación desarrollamos estas ideas, comenzando por el siguiente epígrafe, donde describimos los principales monitores de reputación internaciona- les y el Monitor Español de Reputación Corporativa (MERCO), del que hemos utilizado los datos para el caso español como base de nuestro estudio. Seguidamente analizamos la influencia de distintas variables relacionadas con el desempeño financiero sobre los datos de reputación en España, con el fin de conocer su relación con la reputación corporativa. Después continuamos nuestra discusión y análisis para ver la influencia de la reputación del CD sobre la reputación de la empresa en España. A continuación realizamos un análisis de regresión multivariable imputando las diferentes variables de desempeño financiero y de reputación del CD en el modelo. Finalmente, en el último apartado, realizamos una reflexión sobre los resultados obtenidos.

\section{Monitores de reputación}

A partir de encuestas y mediante diferentes instrumentos de medida, una variedad de organizaciones y medios de comunicación internacionales otorgan anualmente buenas reputaciones a las empresas que lo merecen, constituyendo así los monitores de reputación corporativa. En el Cuadro 1 presentamos los principales monitores de reputación internacionales, así como las organizaciones que los constituyen y alimentan. Cabe destacar que, en general, se trata de organizaciones de medios de comunicación o colaboraciones entre ellos y firmas consultoras internacionales, con la excepción del Reputation Institute, organismo de carácter académico, ubicado en la Facultad de Administración y Dirección de Empresas de la New York University y dirigido por los profesores Charles Fom- 
Papel de la reputación del consejero delegado en la reputación de la empresa española Urra Urbieta, José Anastasio; Mohedano Suanes, Antonia y Safón Cano, Vicente

\section{Cuadro 1}

Principales monitores de reputación corporativa internacionales

\begin{tabular}{lcl}
\hline \multicolumn{1}{c}{ Monitor } & Desde & \multicolumn{1}{c}{ Organización } \\
\hline Global most Admired Companies (GMAC) & 1987 & Fortune, Hay Group \\
Review 200 & 1993 & Fast Eastern Economic Review \\
Britain's most Admired Companies (BMAC) & 1994 & Management Today \\
Reputation Quotient (RQ) & 1999 & $\begin{array}{l}\text { The Wall Street Journal, } \\
\text { The Reputation Institute, }\end{array}$ \\
World's most Respected Companies (WMRC) & 1999 & $\begin{array}{l}\text { Financial Times, } \\
\text { PricewaterhouseCoopers }\end{array}$ \\
\hline
\end{tabular}

Fuente: Elaboración propia.

brum y Cees Van Riel, y cuya misión es "destacar el valor de la reputación corporativa, la gestión de la reputación corporativa, y la medida y valoración de la reputación corporativa".

Como hemos apuntado desde el inicio, la reputación de la empresa se conceptúa como una estimación global de las percepciones en sus principales grupos de interés, o stakeholders, en un marco de comparación entre rivales, de mercado por tanto. La realidad, sin embargo, es que los diferentes stakeholders suelen ser "ayudados" en el desarrollo de su percepciones por las organizaciones tras los monitores de reputación corporativa. Éstas organizaciones, además de evaluadoras de la reputación, se convierten así en prescriptores de la reputación, y, hasta cierto punto, en gestores de reputación corporativa.

Para la elaboración y mantenimiento de los diferentes monitores de reputación corporativa, las organizaciones recurren, como hemos señalado, a estudios soportados por encuestas y diferentes medios de medida. En el Cuadro 2 re- sumimos la metodología e indicadores de los principales monitores de reputación internacionales. El Review 2000, de la Fast Eastern Economic Review, y el $B M A C$ de Management Today, con ligeras diferencias en la muestra o en los ítems utilizados, comparten con el GMAC y el $W M R C$, y a diferencia del $R Q$, la visión de los directivos como único stakeholder y una consideración eminentemente financiera de la reputación.

De forma similar a los monitores de reputación en el contexto internacional, el Monitor Español de Reputación Corporativa (MERCO) es promovido por Villafañe \& Asociados Consultores, el diario Cinco Días y la firma Análisis e Investigación, S. L.; y al igual que sus homólogos internacionales, el MERCO evalúa anualmente desde 2000 la reputación de las principales empresas que operan en España. Con los datos de reputación de cada año se establece un ranking de reputación corporativa de empresas españolas, que se mantiene vigente, a año pasado, hasta que el siguiente y sucesivo estudio es 


\section{Metodología e indicadores de los principales monitores de reputación corporativa internacionales}

\begin{tabular}{|c|c|c|}
\hline Monitor & Metodología & Indicadores \\
\hline $\begin{array}{l}\text { Global most Admired } \\
\text { Companies (GMAC) }\end{array}$ & $\begin{array}{l}\text { Cuestionario a } 10000 \text { directi- } \\
\text { vos y analistas de empresas } \\
\text { de nacionalidad norteameri- } \\
\text { cana, donde se les pide que } \\
\text { consideren una serie de atri- } \\
\text { butos que posteriormente } \\
\text { animan los indicadores de re- } \\
\text { putación corporativa. }\end{array}$ & $\begin{array}{l}\text { 1) Solidez financiera } \\
\text { 2) Valor de las inversiones a largo } \\
\text { plazo } \\
\text { 3) Uso de los activos corporativos } \\
\text { 4) Innovación } \\
\text { 5) Calidad directiva y de la gestión } \\
\text { 6) Calidad de los productos } \\
\text { y servicios } \\
\text { 7) Habilidad para atraer, desarrollar } \\
\text { y mantener el talento } \\
\text { 8) Reconocimiento de la } \\
\text { responsabilidad social } \\
\text { corporativa }\end{array}$ \\
\hline $\begin{array}{l}\text { World's most } \\
\text { Respected Companies } \\
\text { (WMRC) }\end{array}$ & $\begin{array}{l}\text { Cuestionario a } 4000 \text { directi- } \\
\text { vos de } 70 \text { países, donde se } \\
\text { les pide que reflejen las tres } \\
\text { empresas del mundo que } \\
\text { más respeten y las razones } \\
\text { por las que les deparan ese } \\
\text { respeto. Cada año se introdu- } \\
\text { cen cuestiones adicionales } \\
\text { en un intento por depurar el } \\
\text { constructo; así mismo, la in- } \\
\text { formación obtenida de los di- } \\
\text { rectivos se contrasta con } \\
\text { gestores de fondos de inver- } \\
\text { sión, comentaristas de me- } \\
\text { dios de comunicación y res- } \\
\text { ponsables de ONG's. }\end{array}$ & $\begin{array}{l}\text { 1) Fuerte y bien articulada } \\
\text { estrategia } \\
\text { 2) Maximización de la satisfacción } \\
\text { y lealtad de clientes } \\
\text { 3) Liderazgo en el sector } \\
\text { 4) Calidad de los productos } \\
\text { y servicios } \\
\text { 5) Fuerte y consistente beneficio } \\
\text { 6) Cultura corporativa robusta } \\
\text { y humana } \\
\text { 7) Gestión del cambio exitosa } \\
\text { 8) Globalización del negocio }\end{array}$ \\
\hline $\begin{array}{l}\text { Reputation Quotient } \\
\text { (RQ) }\end{array}$ & $\begin{array}{l}\text { Tras varias fases de estudios } \\
\text { piloto, análisis específicos y } \\
\text { grupos de discusión con dife- } \\
\text { rentes stakeholders se aco- } \\
\text { tan } 20 \text { ítems de los que ema- } \\
\text { nan como factores los seis in- } \\
\text { dicadores. }\end{array}$ & $\begin{array}{l}\text { 1) Atractivo emocional } \\
\text { 2) Características de los productos } \\
\text { y servicios } \\
\text { 3) Visión y liderazgo } \\
\text { 4) Entorno de trabajo } \\
\text { 5) Responsabilidad social } \\
\text { y medioambiental } \\
\text { 6) Desempeño financiero }\end{array}$ \\
\hline
\end{tabular}

Fuente: Elaboración propia. 
Papel de la reputación del consejero delegado en la reputación de la empresa española Urra Urbieta, José Anastasio; Mohedano Suanes, Antonia y Safón Cano, Vicente

realizado y publicado ${ }^{3}$. En la Tabla 1 presentamos el ranking de empresas españolas de 2007, confeccionado por el estudio del MERCO con los datos relativos a 2006, y que hemos utilizado como base para nuestro contraste estadístico.

En lo que respecta a su metodología, el estudio del MERCO se encuentra próximo al $R Q$ del Reputation Institute, con el que comparte su consideración plural de los stakeholders, aplicándolo en la recolección de los datos. En sus indicadores, sin embargo, se aproxima más a sus homólogos extranjeros, aunque sin llegar a la preeminencia financiera del GMAC y sin las consideraciones del componente afectivo-emocional del $R Q$. En el Cuadro 3 describimos la metodología e indicadores del MERCO.

Villafañe \& Asociados Consultores, el diario Cinco Días y la firma Análisis e Investigación, S. L. patrocinan además el Monitor Español de Liderazgo Empresarial (MELE). Éste, con las mismas metodología y muestra, también evalúa y establece un ranking anual de reputación de directivos españoles.

\section{Antecedentes de la reputación corporativa: el halo financiero}

Uno de los antecedentes más importantes de la reputación corporativa es el desempeño financiero (Fombrun, 2006). Como ya hemos señalado, algunos monitores de reputación, como el famoso GMAC de Fortune, han sido criticados por estar muy vinculados a las variables de performance financiera ${ }^{4}$, como la rentabilidad, el crecimiento de las ventas, etc., lo que obliga, según Brown \& Perry (1994), a "controlar" esta dimensión en cualquier investigación sobre los antecedentes de la reputación corporativa con el fin de "descontar" el denominado "halo" financiero. Las variables que hemos tenido en cuenta han sido el tamaño de la empresa y la concentración sectorial, como variables que explican la eficiencia; y el crecimiento de las ventas y la rentabilidad, como variables que sintetizan la información contable que reciben los principales stakeholders de una empresa, de forma similar a como han procedido otros investigadores previamente (Fombrun \& Shanley, 1990; McGuire, Schneeweis \& Branch 1990). A continuación estudiamos cada una de ellas.

\section{Tamaño}

El tamaño de la empresa es una variable habitual en el estudio de los antecedentes de la reputación empresarial (De la Fuente Sabaté \& Puente, 2003b), habiéndose observado en numerosas ocasiones una relación positiva estadísticamente significativa entre

3 El ranking no es exactamente de empresas españolas, sino de empresas que operan en España, puesto que entre las 100 empresas más reputadas el MERCO incluye algunas filiales españolas de multinacionales extranjeras, como son los casos de Accenture, Adecco, Alcampo o Amadeus, entre otras.

4 McGuire, Schneeweis \& Branch (1990), y Fombrun \& Shanley (1990) comprobaron que las variables financieras explicaban gran parte de la varianza del ranking de reputación publicado por Fortune. 
Tabla 1

Ranking de empresas españolas 2007

\begin{tabular}{|c|c|c|c|}
\hline Empresa & Puntuación & Empresa & Puntuación \\
\hline EL CORTE INGLES & 10.000 & LECHE PASCUAL & 2.850 \\
\hline INDITEX & 9.980 & BSH ELECTRODOMESTICOS & 2.844 \\
\hline GRUPO TELEFONICA & 8.854 & ERICSSON & 2.810 \\
\hline REPSOL YPF & 7.304 & NH HOTELES & 2.810 \\
\hline BBVA & 7.228 & ISOFOTON & 2.796 \\
\hline SANTANDER & 7.224 & AC HOTELES & 2.786 \\
\hline LA CAIXA & 6.792 & ESADE & 2.780 \\
\hline IBERDROLA & 5.880 & SANITAS & 2.778 \\
\hline ENDESA & 5.028 & ING DIRECT & 2.674 \\
\hline FERROVIAL & 4.896 & ONCE-FUNDOSA & 2.664 \\
\hline MERCADONA & 4.738 & GRUPO PRISA & 2.638 \\
\hline CAJA MADRID & 4.724 & CARREFOUR & 2.618 \\
\hline INDRA & 4.390 & BP OIL & 2.614 \\
\hline MAPFRE & 4.370 & GENERAL ELECTRIC & 2.612 \\
\hline ACCIONA & 4.368 & SOS CUETARA & 2.568 \\
\hline VODAFONE & 4.304 & HOLCIM & 2.458 \\
\hline SIEMENS & 4.092 & VOCENTO & 2.454 \\
\hline BANKINTER & 4.058 & PROSEGUR & 2.450 \\
\hline ACS & 4.056 & RED ELECTRICA DE ESPAÑA & 2.418 \\
\hline BANCO POPULAR & 3.946 & EBRO PULEVA & 2.346 \\
\hline IBM & 3.942 & L'OREAL & 2.324 \\
\hline GAS NATURAL & 3.930 & MANGO & 2.312 \\
\hline MICROSOFT & 3.818 & TARRADELLAS & 2.286 \\
\hline ABERTIS & 3.738 & GRUPO PLANETA & 2.282 \\
\hline GRUPO AGBAR & 3.702 & $\begin{array}{l}\text { MONDRAGON CORPORACION } \\
\text { COOPERATIVA }\end{array}$ & 2.202 \\
\hline GAMESA & 3.696 & HEWLETT PACKARD & 2.058 \\
\hline ACCENTURE & 3.630 & ADESLAS & 1.998 \\
\hline IBERIA & 3.622 & ESIC & 1.758 \\
\hline NOVARTIS & 3.608 & SONY & 1.632 \\
\hline INSTITUTO DE EMPRESA & 3.570 & LILLY & 1.626 \\
\hline IESE & 3.538 & CATALANA OCCIDENTE & 1.624 \\
\hline NESTLE & 3.374 & MUTUA MADRILEÑA & 1.564 \\
\hline
\end{tabular}


Papel de la reputación del consejero delegado en la reputación de la empresa española Urra Urbieta, José Anastasio; Mohedano Suanes, Antonia y Safón Cano, Vicente

Tabla 1

Ranking de empresas españolas 2007(Continuación)

\begin{tabular}{llll}
\hline Empresa & Puntuación & Empresa & Puntuación \\
\hline MRW & 3.346 & $\begin{array}{l}\text { CLINICA UNIVERSITARIA } \\
\text { DE NAVARRA }\end{array}$ & 1.554 \\
BANESTO & 3.344 & PROCTER \& GAMBLE & 1.550 \\
PRICEWATERHOUSECOOPERS & 3.328 & TOYOTA & 1.514 \\
DANONE & 3.326 & EAE-ESCUELADE ADMINISTRACION & 1.498 \\
& & DE EMPRESAS & \\
DKV SEGUROS & 3.312 & METROVACESA & 1.496 \\
CEPSA & 3.270 & ABENGOA & 1.470 \\
GRUPO EROSKI & 3.246 & CLH & 1.460 \\
UNION FENOSA & 3.236 & GOOGLE & 1.448 \\
BANCO SABADELL ATLANTICO & 3.168 & CAIXA CATALUNYA & 1.436 \\
COCA COLA & 3.158 & ONO & 1.422 \\
BANCAJA & 3.152 & ADECCO & 1.420 \\
DELOITTE & 3.122 & RENAULT & 1.390 \\
NOKIA & 3.078 & CAMPOFRIO & 1.318 \\
ORANGE & 3.066 & ASLAND LAFARGE & 1.292 \\
FCC & 2.972 & SOL MELIA & 1.270 \\
IKEA & 2.972 & ELTIA & 1.252 \\
RENFE & 2.952 & BASF & 1.240 \\
SACYR - VALLEHERMOSO & 2.914 & UNICAJA & 1.213 \\
\hline FUE: MERCO. & & &
\end{tabular}

Fuente: MERCO.

estas dos variables. El tamaño se relaciona con mejoras en la eficiencia derivadas de las mayores economías de escala, lo que permite mejorar la rentabilidad y por ende la reputación; además, ayuda a una empresa a ser notoria, pues más gente interactúa con ella y más presente está en los medios, lo que también mejora su reputación (Fombrun \& Shanley, 1990). Es tal la relación entre tamaño y reputación, que algunos trabajos han utilizado el tamaño como medida supletoria, o proxy, de la reputación en sus estudios empíri- cos (por ejemplo, Akabayashi, FujiwaraGreve \& Greve, 2005).

En la Tabla 2 se observa que, para estar en el ranking de empresas más reputadas del MERCO, hay que ser grande, lo que parece ser una condición necesaria para entrar en este ranking y en otros análogos (por ejemplo, el de Fortune).

La columna $A^{5}$ recoge el promedio de ventas, activos, empleados, ratio de concentración sectorial en los años 2003 a 2005 , crecimiento de las ventas y rentabilidad de los activos de las empresas del 


\section{Cuadro 3 \\ Metodología e indicadores del monitor de reputación corporativa MERCO}

\begin{tabular}{|c|c|}
\hline Metodología & Indicadores \\
\hline $\begin{array}{l}\text { El estudio del MERCO se desarrolla anualmente desde } \\
2000 \text {. El estudio se realiza en el 3er trimestre de cada año; el } \\
\text { ranking se publica durante el 1er trimestre del año siguiente } \\
\text { a su elaboración, y permanece vigente durante todo ese año } \\
\text { posterior, hasta la publicación del siguiente ranking. La me- } \\
\text { todología del estudio se realiza en } 3 \text { fases: } \\
\text { 1) Cuestionario a una muestra significativa (error 2'5\%, nivel } \\
\text { de confianza 95'5 y p=q) de directivos de empresas que ope- } \\
\text { ran en España y cuya facturación supera los } 45 \text { millones de } \\
\text { euros. A los encuestados se les solicita una lista de empresas } \\
\text { con mejor reputación general y otra de su sector, que constitu- } \\
\text { yen la base para la confección de un ranking provisional. } \\
\text { 2) Cada empresa del ranking provisional es evaluada: } \\
\text { a. Por una muestra de analistas financieros del Instituto Es- } \\
\text { pañol de Analistas Financieros (IEAF). } \\
\text { b. Por una muestra de dirigentes de ONG's. } \\
\text { c. Por una muestra de representantes sindicales. } \\
\text { d. Por una muestra de asociaciones de consumidores. } \\
\text { e. Mediante el análisis de fuentes secundarias suministradas } \\
\text { por cada empresa. } \\
\text { f. A través de un cuestionario de méritos in situ por un técnico } \\
\text { del MERCO. } \\
\text { 3) Con el doble análisis se confecciona el ranking anual defi- } \\
\text { nitivo. }\end{array}$ & $\begin{array}{l}18 \text { ítems que se agrupan de } \\
\text { tres en tres para formar } 6 \\
\text { factores o indicadores: } \\
\text { 1) Resultados económico- } \\
\text { financieros } \\
\text { 2) Calidad de productos y } \\
\text { servicios } \\
\text { 3) Cultura corporativo y calidad } \\
\text { laboral } \\
\text { 4) Ética y Responsabilidad } \\
\text { Social Corporativa } \\
\text { 5) Dimensión global y presencia } \\
\text { internacional } \\
\text { 6) Innovación }\end{array}$ \\
\hline
\end{tabular}

Fuente: Elaboración propia a partir del MERCO.

5 La mayoría de las medias se han calculado sobre una muestra de 73 empresas. La razón de este proceder ha estado en la exclusión de ciertas empresas del ranking del MERCO de 2007. Así, hemos excluido las escuelas de negocio ya que la base de datos de Bureau van Dijk, Sistema de Análisis de Balances Ibéricos (SABI), que hemos utilizado para la extracción y cálculo de las variables financieras y de agregación sectorial, carece de datos sobre este tipo de entidades, y no nos ha sido posible acceder a los datos para estas empresas; tampoco hemos podido acceder a los datos relativos a entidades aseguradoras; además, tras investigar todas y cada una de las 100 empresas del ranking, hemos excluido las empresas filiales de otras empresas ya presentes en el ranking, dado que incluirlas, al formar grupo, supondría una duplicación de puestos y puntuaciones. Para la extracción y cálculo de las variables financieras y de agregación sectorial relativas a las entidades de crédito del ranking hemos utilizado la base de datos de entidades de crédito de INTERTELL. 
Papel de la reputación del consejero delegado en la reputación de la empresa española Urra Urbieta, José Anastasio; Mohedano Suanes, Antonia y Safón Cano, Vicente

Tabla 2 Caracterización de las empresas del ranking del MERCO

\begin{tabular}{lccc}
\hline & $\begin{array}{c}\text { A } \\
\text { Empresas del ranking } \\
\text { del MERCO 2007 (media) }\end{array}$ & $\begin{array}{c}\text { B } \\
\text { Promedio sectorial } \\
\text { (SIC principal) }\end{array}$ & A/B \\
\hline Ventas_2005 (x1000) & $4.585 .739,53$ & $181.023,09$ & 25,33 \\
Ventas_2004 (x1000) & $3.949 .590,48$ & $143.662,89$ & 27,49 \\
Ventas_2003 (x1000) & $3.655 .549,29$ & $131.013,90$ & 27,90 \\
Activo_2005 (x1000) & $23.605 .656,56$ & $1.515 .615,67$ & 15,57 \\
Activo_2004 (x1000) & $18.169 .974,35$ & $1.192 .781,03$ & 15,23 \\
Activo_2003 (x1000) & $16.380 .937,07$ & $1.054 .955,74$ & 15,53 \\
Empleados_2005 & $13.826,49$ & 341,75 & 40,46 \\
Empleados_2004 & $15.027,70$ & 333,14 & 45,11 \\
Empleados_2003 & $14.221,47$ & 325,46 & 43,70 \\
CR5_05 & N/A & 49,51 & N/A \\
CR10_05 & N/A & 58,87 & N/A \\
Cto_Ventas_en_2005 & 11,86 & 12,34 & 0,96 \\
Cto_Ventas_en_2004 & 12,95 & 10,12 & 1,28 \\
Cto_Ventas_en_2003 & 8,66 & 9,14 & 0,95 \\
ROA_2005 & 7,13 & 2,90 & 2,46 \\
ROA_2004 & 7,71 & 3,42 & 2,25 \\
ROA_2003 & 6,83 & 3,68 & 1,86 \\
\hline
\end{tabular}

Nota: La mayoría de las medias se han calculado sobre una muestra de 73 empresas. La variable "Empleados" es la que menos muestra ha utilizado, con un mínimo de 59 empresas el año 2003.

ranking del MERCO publicado en 2007. La columna $\mathrm{B}^{6}$ ilustra los mismos datos, pero para el promedio sectorial obtenido de las empresas del mismo SIC (Standard Industrial Classification) que el SIC principal de las empresas del listado del MERCO. Finalmente, la columna $A / B$ es una medida relativa del tamaño y rentabilidad de las "empresas MERCO" en comparación con su grupo de referencia. En promedio, las empresas que lista el MERCO fueron, en 2005, entre 40, 25 y 15 veces más grandes que las de sus sectores de referencia, si se consideran los em-

6 Para los cálculos de los promedios sectoriales hemos utilizado la base de datos de Bureau van Dijk, Sistema de Análisis de Balances Ibéricos (SABI), excepto para el sector de entidades de crédito, cuyos datos hemos extraído de la base de datos de entidades de crédito de INTERTELL. 
pleados, las ventas y los activos, respectivamente, lo que pone de manifiesto la importancia del tamaño.

Ser grande parece ser una condición necesaria para estar presente en el ranking del MERCO, y ser muy grande parece ser una condición necesaria para estar en los primeros puestos del ranking. La Tabla 3 muestra, entre otras, la correlación entre las ventas de las empresas del ranking del MERCO en 2007, como indicador el tamaño, y el valor de su "reputación". Se aprecia una relación positiva intensa y significativa, lo que significa que para estar en los primeros puestos del ranking, hay que tener un gran tamaño. De estas conclusiones derivamos la primera hipótesis de este trabajo:

$\mathbf{H}_{1}$ : Cuanto mayor sea su tamaño, mayor será el nivel de reputación de las empresas incluidas en el ranking del MERCO.

\section{Crecimiento y rentabilidad}

El crecimiento y la rentabilidad son dos importantes antecedentes de la reputación de una empresa (Fombrun, 2006), pues si son altos predisponen favorablemente a los stakehoders de una empresa (Fombrun \& Shanley, 1990). A tenor de los datos de reputación del MERCO, en España, el crecimiento de las ventas no es una variable determinante de la reputación de una empresa, pues, de los tres años considerados, en dos de ellos las empresas del listado del MERCO crecieron menos que el promedio sectorial, y en el año que lo superaron, lo hicieron tímidamente. Tampoco parece que esta variable de performance explique la posición en el ranking, pues, como se aprecia en la Tabla 3, el crecimiento de las ventas y el valor de la reputación obtenido por el
MERCO presentan una correlación no significativa.

La rentabilidad de los activos es más o menos el doble en las empresas del ranking del MERCO que en el promedio sectorial (Tabla 2, ROA), lo que parece ser una prueba de que, también para el caso de España, la rentabilidad está asociada con la reputación. No obstante, ya que el tamaño produce mayor eficiencia, y ésta mejores resultados, es posible que la relación anterior sea espuria, y que la asociación entre rentabilidad y reputación esté explicada por la relación entre el tamaño y la reputación, y el tamaño y la rentabilidad. Esta hipótesis cobra fuerza si observamos la Tabla 3 , en la que se muestra una correlación no significativa entre el promedio de ROAs de los años 2003-05 y la puntuación recibida por las empresas del estudio del MERCO en la variable reputación.

Se concluye de estos análisis preliminares que, en España, ni el crecimiento de las ventas ni la rentabilidad explican la inclusión de una empresa en el ranking del MERCO, ni su posición en el mismo. En la sección 5 indagaremos más sobre esta cuestión, testando la siguiente hipótesis:

$\mathbf{H}_{2}$ : Ni el crecimiento de las ventas $\left(\mathbf{H}_{2 \mathrm{a}}\right)$ ni la rentabilidad $\left(\mathbf{H}_{2 \mathrm{~b}}\right)$ de la empresa están relacionados con el nivel de reputación de las empresas incluidas en el ranking del MERCO.

\section{Concentración sectorial}

La concentración sectorial puede ser una importante característica estructural que influya en la reputación de la empresa, ya sea porque está relacionada con mayores beneficios, fruto de la colusión (véase esta explicación, propuesta por la Economía Industrial, en Basdeo, 
Papel de la reputación del consejero delegado en la reputación de la empresa española Urra Urbieta, José Anastasio; Mohedano Suanes, Antonia y Safón Cano, Vicente

Tabla 3

Matriz de correlaciones ${ }^{8}$

\begin{tabular}{|c|c|c|c|c|c|c|}
\hline & 1. & 2. & 3. & 4. & 5. & 6. \\
\hline 1. In_Firmrep_07 & 1 & & & & & \\
\hline 2. In_Ceorep_07 &, $81\left(^{* *}\right)$ & 1 & & & & \\
\hline 3. In_Ventas_05 &, $62\left(^{* *}\right)$ &, $55\left(^{* *}\right)$ & 1 & & & \\
\hline 4. CR5_05 &, $41\left(^{* *}\right)$ & ,20 &, $46\left({ }^{* *}\right)$ & 1 & & \\
\hline 5. Cto_Ventas_relativo_03_05 & 14 &,- 02 &,- 07 & ,02 & 1 & \\
\hline 6. ROA_relativo $03 \_05$ & 15 &,- 09 & ,18 &, $25\left(^{*}\right)$ & ,13 & 1 \\
\hline
\end{tabular}

$\mathrm{N}=73$; ${ }^{*}$ Significativa al nivel 0,01 (bilateral); * Significativa al nivel 0,05 (bilateral).

Smith, Grimm, Rindova \& Derfus, 2006), o porque incrementa la probabilidad de conocer la empresa ${ }^{7}$, y por tanto la probabilidad de que la empresa sea notoria, una dimensión fundamental de la reputación (Rindova, Williamson, Petkova \& Sever, 2005).

Las empresas que aparecen en el ranking del MERCO están en sectores muy concentrados pues, como se recoge en la Tabla 2, en promedio, la suma de las cuotas de mercado de las cinco y diez empresas más grandes suponen aproximadamente el $50 \%$ y $60 \%$ del total del sector, respectivamente. Este nivel de concentración sectorial es muy superior al que encontramos en la mayor parte de sectores de la economía española, lo que nos deja entrever una probable asociación entre concentración del sector y reputación empresarial. Los datos de correlación de la Tabla 3 avalan esta asunción, pues, ya dentro del ranking del MERCO, las empresas que están en los sectores más concentrados son las que más reputación consiguen, esto es, más altas están en el ranking. A la vista de estos resultados proponemos la siguiente hipótesis:

$\mathrm{H}_{3}$ : Cuanto mayor sea el grado de concentración sectorial, mayor será el nivel de reputación de las empresas incluidas en el ranking del MERCO.

7 La concentración sectorial es un indicador del tamaño relativo de las empresas de un sector en relación con el propio sector, y es función del número de empresas del sector y de sus respectivas cuotas de mercado, aumentando cuando el número de empresas es más reducido y sus cuotas de mercado mayores. Así pues, una alta concentración sectorial implica un menor número de empresas y mayores tamaños de las mismas, lo que incrementa la probabilidad de que sean más notorias.

8 Los cálculos de regresión múltiple y el ajuste del modelo objeto de contraste se han realizado para una muestra de 73 empresas (véase la nota 5). Respecto de las variables que configuran el modelo objeto de contraste, nos hemos distanciado de la Recomendación de la Comisión de las Comunidades Europeas (2003/361/CE), optando por la variable "Ventas" para imputar el tamaño por dos motivos: 1) la correlación entre la variable "Ventas" y la variable "Activos" es casi perfecta, y 2) la dificultad de obtener los datos relativos a la variable "Empleados" para todas las empresas de la muestra. 


\section{Influencia de la reputación del CEO sobre la reputación de la empresa}

La reputación del $C E O$, o Consejero Delegado, es una señal que influye en la mayoría de los stakeholders de una empresa (Solomon, Reckers \& Lowe, 2005; Mazur, 1999), aunque lo hace de forma especial entre inversores y empleados (Helm, 2006). Hoffman (1999) afirma que el CEO pone rostro a la empresa y es clave para la configuración de su reputación, y Garbett (1988) entiende que la personalidad y el estilo del CEO es el factor más importante en la formación de la personalidad e imagen de la empresa.

Uno de los problemas más difíciles de resolver cuando se aborda el estudio de la relación entre la reputación del CD y su empresa es la determinación de la dirección causal entre ambas variables (Hutton, Goodman, Alexander \& Genest, 2001). A pesar de que la literatura sostiene de forma general que la reputación del CD influye en la reputación de la empresa, el sentido común obliga a considerar también la relación inversa, e incluso recíproca, pues no es muy descabellado pensar que una empresa con una gran reputación que contrata a un nuevo CEO poco conocido, influirá positivamente en la reputación de éste. En este trabajo analizamos la reputación del CEO como causa, pero debe advertirse que esta variable puede ser también efecto.

De acuerdo con el informe Corporate Reputation Watch (Harris Interactive, 2002), realizado, como hemos señalado, sobre una muestra de 800 Chief Executie Officers (CEO's) y directivos de USA, Canadá, Bélgica, Italia, Alemania, Reino Uni- do y Holanda, la reputación del CEO supone entre el $30 \%$ y el $65 \%$ de la reputación de la empresa, según el país considerado. En España, según el informe La Gestión Empresarial en los Años 2005/06, elaborado por la consultora Burson-Marsteller, el $38,4 \%$ de los empresarios encuestados consideraron que entre un $50 \%$ y un $90 \%$ de la reputación de una empresa respondía a la de su Consejero Delegado, lo que confirma la importancia de esta variable sobre la reputación de la empresa.

La Tabla 3 muestra una fuerte correlación entre las reputaciones del CEO y de la empresa, lo que refuerza la idea de una relación causal entre la reputación del CD o CEO y la reputación de la empresa, estimada en el $0,81\left(R^{2}=65,61 \%\right)$, lo que nos permite proponer la siguiente hipótesis:

$\mathbf{H}_{4}$ : Cuanto mayor sea la reputación de su Consejero Delegado, mayor será la reputación de las empresas incluidas en el ranking del MERCO.

\section{Resultados}

Con el fin de estudiar las hipótesis planteadas en la sección anterior, sometimos los datos del MERCO a un análisis de regresión múltiple, asumiendo una relación funcional lineal entre la reputación de la empresa y las variables reputación del CD, tamaño, concentración del sector, crecimiento de las ventas y rentabilidad, según el siguiente modelo:

Firmrep $(\mathbf{t})=\mathrm{f}[$ Ceorep $(\mathrm{t})$, Ventas $(\mathrm{t}-1), \mathrm{CR} 5$ (t-1), Cto_Ventas_relativo (t-x), ROA_relativo $(\mathrm{t}-\mathrm{x})]$

Donde:

$\mathrm{t}=2006$ (Ranking 2007)

Firmrep $=$ Log neperiano de la reputación corporativa 
Papel de la reputación del consejero delegado en la reputación de la empresa española Urra Urbieta, José Anastasio; Mohedano Suanes, Antonia y Safón Cano, Vicente

Ceorep $=$ Log neperiano de la reputación del CD o CEO

Ventas = Log neperiano de las ventas, como medida del tamaño

CR5 = Concentración en el sector, medida con el CR5.

Cto_Ventas_relativo $=$ promedio del crecimiento de las ventas en $\mathrm{t}-1, \mathrm{t}-2$ y $\mathrm{t}-3$

$\mathrm{ROA}$ _relativo $=$ promedio de la rentabilidad sobre los activos en $\mathrm{t}-1, \mathrm{t}-2$ y t-3

La Tabla 4 muestra los resultados del análisis. El primer modelo regresa la reputación de la empresa sobre las variables que hemos considerado como variables de control del efecto "halo" financiero, mientras que el segundo introduce, además, la reputación del CD como principal variable independiente de estudio.

El Modelo 2 ajusta bien los datos ( $R$ cuadrado corregida $=0,74$ ), y supone una mejora significativa en su capacidad predictiva frente al Modelo 1 (incremento significativo en el $\mathrm{R}$ cuadrado), además de no presentar problemas de multicolineali- dad (FIV máximo 10, según Hair, Anderson, Tatham \& Black, 1998).

En este modelo, considerando un nivel de confianza de 0,05 , son significativamente diferentes de cero los parámetros asociados a las variables grado de concentración sectorial, crecimiento de las ventas y reputación del CD o CEO. Estos resultados permiten aceptar las hipótesis $\mathrm{H}_{2 \mathrm{~b}}, \mathrm{H}_{3}$ y $\mathrm{H}_{4}$; mientras que nos llevan a rechazar las hipótesis $\mathrm{H}_{1}$ y $\mathrm{H}_{2 a}$.

El tamaño, medido con las ventas en nuestros análisis, es la variable de mayor impacto sobre la reputación en el Modelo 1, aunque deja de serlo en el Modelo 2 , tras la inclusión de la reputación del CEO. Por el contrario, el crecimiento y la concentración no son significativas en el Modelo 1, y sí lo son en el Modelo 2. La explicación a este hecho radica en las correlaciones entre las variables. La introducción de la reputación del CD anula el impacto del tamaño sobre la reputación de la empresa, pues su correlación con la

Tabla 4

Resultados del análisis de regresión múltiple

\begin{tabular}{lcccc}
\hline & \multicolumn{2}{c}{ Modelo 1 } & \multicolumn{2}{c}{ Modelo2 } \\
\hline Dependiente: Reputación de la empresa & Coef. estand. & Signif. & Coef. estand. & Signif. \\
\hline Constante & & 0,00 & & 0,00 \\
In_Ventas_05 & 0,57 & 0,00 & 0,13 & 0,14 \\
CR5_05 & 0,14 & 0,22 & 0,17 & 0,03 \\
Cto_Ventas_relativo_03_05 & 0,18 & 0,08 & 0,14 & 0,03 \\
ROA_relativo_03_05 & $-0,01$ & 0,89 & 0,13 & 0,07 \\
In_Ceorep07 & & & 0,71 & 0,00 \\
& & & & \\
R cuadrado & 0,66 & & 0,76 & \\
R cuadrado corregida & 0,43 & & 0,74 & \\
FIV máximo & 1,33 & & 1,86 & \\
\hline
\end{tabular}

Nota: estimaciones realizadas con regresión múltiple por el método de mínimos cuadros ordinarios.

Fuente: Elaboración propia. 
variable dependiente es mayor $(0,81$ frente a 0,62 ) y entre estos dos predictores hay una fuerte asociación (correlación de 0,55). Al quedar excluido el tamaño, entran algunas variables que antes no eran significativas porque están menos correlacionadas con la reputación del CD que con el tamaño, y presentan correlaciones con la variable dependiente de menor intensidad que las que tenían con el tamaño.

\section{Conclusiones}

En este artículo hemos justificado la importancia de la reputación corporativa como recurso intangible fuente de ventajas competitivas, y hemos analizado algunos antecedentes de este constructo en una muestra de empresas españolas.

En particular, hemos observado la influencia de la reputación del CEO manteniendo bajo control estadístico cuatro variables descriptoras del llamado halo financiero.

Tras proponer varias hipótesis derivadas de la literatura y de un primer análisis univariante de los datos manejados, hemos realizado un análisis multivariable. De los análisis practicados y los resultados se desprenden diferentes conclusiones circunscritas al caso analizado, y otras de alcance más general.

En lo que respecta al caso analizado, el Monitor Español de Reputación Corporativa, en primer lugar, para estar presente en su ranking, el tamaño es una variable fundamental, lo que puede verse como una condición necesaria. Por otra parte, para entrar en el ranking del MERCO no es muy importante crecer por encima del promedio sectorial, pero sí parece necesario ser más rentable que el promedio del sector. Además, una vez las empresas entran en el ranking, lo importante ya no es el tamaño, sino la reputación del CEO y la visibilidad que proporciona estar en sectores concentrados; por lo que la mayor o menor valoración que los stakeholders hacen de la reputación corporativa no depende de la performance financiera ni del tamaño (en esta parte del estudio medido con las ventas).

Estas conclusiones conllevan implicaciones para las empresas. Si la reputación genera rentas extraordinarias, las empresas deben saber que, para entrar en el ranking del MERCO deben ser grandes. Estrategias como las fusiones y adquisiciones son las que de forma más rápida conducen al crecimiento corporativo, siendo además estrategias que permiten la concentración industrial si se realizan con competidores, lo cual permitirá "entrar en el ranking" y "escalar en el ranking", ya que la concentración se ha manifestado como un predictor de la reputación. Además, si una empresa ya está en el ranking y desea mejorar su puesto, puede conseguirlo mejorando la reputación de su CD, contratando, por ejemplo, a un CD con reputación o invirtiendo dinero en su imagen pública.

En lo que respecta al alcance general de nuestro estudio, la realidad prácticamente nos ha ido tomando la delantera, al mostrarnos, tal como se desprende de nuestros resultados, la intensa relación existente entre la reputación del CEO y la reputación corporativa. Efectivamente, los acontecimientos que han venido desarrollándose en el sistema financiero, y que han servido de catalizador del actual panorama de aguda crisis 
Papel de la reputación del consejero delegado en la reputación de la empresa española Urra Urbieta, José Anastasio; Mohedano Suanes, Antonia y Safón Cano, Vicente

mundial que padecemos, nos han hecho ver como la avaricia, la visión a corto plazo y, en general, los comportamientos carentes de toda ética de muchos CEOs y directivos, no sólo han destruido su reputación, sino también la de las empresas que dirigían y animaban, extendiéndose esa destrucción, en muchos casos, a las propias empresas en su totalidad. Estos directivos han dilapidado su reputación y la de sus empresas, y las que todavía se mantienen activas han quedado seriamente dañadas por la pérdida de confianza en ellas por parte de los stakeholders, extendiéndose además esta desconfianza a todo el sistema financiero y a todo el mundo en una especie de "efecto dominó". Si, como la realidad y nuestros resultados ponen de manifiesto, la reputación de la empresa depende en gran medida de la reputación de sus directivos, la cuestión surge inmediatamente: ¿de qué depende la reputación del CEO?

Esta cuestión abre una muy oportuna, útil y prometedora línea de investigación: los antecedentes de la reputación del CEO. Sin embargo, varias cuestiones deben ser consideradas.

Por una parte, ¿cómo medir la reputación del CEO? Los monitores existentes lo hacen, pero limitándose a sus muestras. También pueden utilizarse escalas validadas para una medida de reputación basada en la personalidad (Davies, Chun, Vinhas da Silva \& Roper, 2001, 2003), con lo que las limitaciones de la muestra quedarían a expensas del diseño de la investigación.

De otro lado, es pertinente separar comportamientos por omisión y comportamientos por acción en la búsqueda de los antecedentes de la reputación del CEO. La reputación no sólo varía por "hacer", sino también por "no hacer".

Además, entre los antecedentes de la reputación del CEO ineludiblemente debe explorarse el componente ético, y la consideración y el alcance de las externalidades.

Finalmente, y para terminar, no podemos dejar de reconocer las limitaciones de este estudio. En este sentido, la principal limitación que observamos es la hipótesis de relación-dirección causal entre reputación del CD y reputación corporativa. Como ya hemos señalado, a pesar de que la literatura sostiene de forma general que la reputación del CD influye en la reputación de la empresa, el sentido común obliga a considerar también la relación inversa, e incluso recíproca, pues no es muy atrevido pensar que una empresa con una gran reputación que contrata a un nuevo CEO poco conocido influirá positivamente en la reputación de éste. Sin embargo, este pensamiento no resulta tan lógico a la inversa: una empresa con una mala reputación que contrata a un nuevo CEO con una gran reputación no tiene por qué influir negativamente en la reputación de éste. En cualquier caso, esta idea requiere ser explorada adicionalmente, aunque quizás la realidad nos tome también la delantera en esto.

\section{Referencias Bibliográficas}

Akabayashi, Hideo; Fujiwara-Greve, Takako y Greve, Henrich (2005). Effects on reputation and family on labour turnover: evidence from Japanese data. Working Paper, Department of Economics, Keio University. 
Barnett, Michael; Jermier, John y Lafferty, Barbara (2006). Corporate Reputation: The Definitional Landscape. Corporate Reputation Review, Vol. 9, No 1, pp 26-38.

Basdeo, Dax; Smith, Ken; Grimm, Curtis; Rindova, Violina y Derfus, Pamela (2006). The impact of market actions on firm reputation. Strategic Management Journal, Vol. 27, No 12, pp 1205-1219.

Brown, Brad y Perry, Susan (1994). Removing the financial performance halo from Fortune's most admired companies. Academy of Management Journal, Vol. 37, No 5, pp 1347-1359.

Chun, Rosa (2005). Corporate reputation: Meaning and measurement. International Journal of Management Reviews, Vol. 7, No 2, pp 91-109.

Comisión de las Comunidades Europeas CE (2003). Recomendación de la Comisión de las Comunidades Europeas (2003/361/CE) de 6 de mayo de 2003 sobre la definición de microempresas, pequeñas y medianas empresas, Bruselas.

Davies, Gary; Chun, Rosa; Vinhas da Silva, Rui y Roper, Stuart (2001). The personification metaphor as a measurement approach for corporate reputation. Corporate Reputation Review, Vol. 4, No 2, pp 113-127.

Davies, Gary; Chun, Rosa; Vinhas da Silva, Rui y Roper, Stuart (2003). Corporate Reputation and Competitiveness. London, England. Editorial Routledge.

De la Fuente Sabaté, Juan Manuel y De Quevedo Puente, Esther (2003a). The concept and measurement of corporate reputation: an application to Spanish financial intermediaries. Corporate Reputation Review, Vol. 5, No 4, pp 280-302.

De la Fuente Sabaté, Juan Manuel y De Quevedo Puente, Esther (2003b). Empiri- cal analysis of the relationship between corporate reputation and financial performance: A survey of the literature. Corporate Reputation Review, Vol. 6, No 2, pp 161-177.

Dhalla, Rumina y Carayannopoulos, Sofy (2006). Understanding when stakeholders discount reputations, 10th Anniversary Conference on Reputation, Image, Identity and Competitiveness, 25-28 May, New York.

Fombrun, Charles (2006). The Rep Track System. 10th Anniversary Conference on Reputation, Image, Identity and Competitiveness, 25-28 May, New York.

Fombrun, Charles y Gardberg, Naomi (2000), Who's Top in Corporate Reputation? Corporate Reputation Review, Vol. 3, No 1, pp 13-17.

Fombrun, Charles; Gardberg, Naomi \& Sever, Joy (2000). The Reputation Quotient: A multi-stakeholder measure of corporate reputation. Journal of Brand Management, Vol. 7, No 4, pp 241-255.

Fombrun, Charles y Shanley, Mark (1990). What's in a Name? Reputation Building and Corporate Strategy. Academy of Management Journal, Vol. 33, No 2, pp 233-258.

Gaines-Ross, Leslie (2000), CEO Reputation: A Key Factor in Shareholder Value. Corporate Reputation Review, Vol. 3, No 4, pp 366-370.

Garbett, Thomas (1988). How to Build a Corporation's Identity and Project Its Image. Massachusetts. Editorial Lexington.

Gotsi, Manto y Wilson, Alan (2001). Corporate reputation: seeking a definition. Corporate Communications, Vol. 6 , No 1, pp 24-30.

Grupp, Robert y Gaines-Ross, Leslie (2002). Reputation management in the biotechnology industry. Journal of Commercial Biotechnology, Vol. 9, No 1, pp 17-26. 
Papel de la reputación del consejero delegado en la reputación de la empresa española Urra Urbieta, José Anastasio; Mohedano Suanes, Antonia y Safón Cano, Vicente

Hair, Joseph; Anderson, Rolph; Tatham, Ronald y Black, William (1998). Multivariate Data Analysis. ( $5^{\text {th }}$ edition). Upper Saddle River. Editorial Prentice-Hall.

Hall, Richard (1992). The Strategic Analysis of Intangible Resources. Strategic Management Journal, Vol. 13, No 2, pp 135-144.

Harris Interactive (2002). Harris Interactive Fourth Annual Corporate Reputation Report: CEOs on Corporate Reputation Influencers, Corporate Social Responsibility, and BoardLevel Involvement, Hill \& Knowlton.

Helm, Sabrina (2005). Designing a Formative Measure for Corporate Reputation. Corporate Reputation Review, Vol. 8, No 2, pp 95-109.

Helm, Sabrina (2006). Common grounds in the perception of corporate reputation? A comparison of three stakeholder groups. 10th Anniversary Conference on Reputation, Image, Identity and Competitiveness, 25-28 May, New York.

Hoffman, Lou (1999). Reputation starts with the CEO. MC Technology Marketing Intelligence, Vol. 19, No 7, pp 56-58.

Hutton, James (1999). The definition, dimensions, and domain of public relations. Public Relations Review, Vol. 25, No 2, pp 199-214.

Hutton, James; Goodman, Michael; Alexander, Jill y Genest, Christina (2001). Reputation management: The new face of corporate public relations? Public Relations Review, Vol. 27, No 3, pp 247-261.

Kranton, Rachel (1996). The Formation of Cooperative Relationships. Journal of Law, Economics \& Organization, Vol. 12, No 1, pp 214-233.

Mazur, Laura (1999). Time to buff the chief executive's global charisma. Marketing, Vol.18, pp 20.
McGuire, Jean; Schneeweis, Thomas y Branch, Ben (1990). Perceptions of firm quality: A cause or result of firm performance. Journal of Management, Vol. 16, No 1, pp 167-180.

McGuire, Jean; Sundgren, Alison y Schneeweis, Thomas (1988). Corporate Social Responsibility and Firm Financial Performance. Academy of Management Journal, Vol. 31, No 4, pp 854-872.

Nisbett, Richard y Ross, Lee (1980). Human Inference: Strategies and Shortcomings of Social Judgment. New Jersey. Editorial Prentice-Hall.

Rindova, Violina; Williamson, lan Oliver; Petkova, Antoaneta y Sever, Joy Marie (2005). Being Good or Being Known: An Empirical Examination of the Dimensions, Antecedents, and Consequences of Organizational Reputation. Academy of Management Journal, Vol. 48, No 6, pp 1033-1049.

Rodríguez Carrasco, José Manuel (2004). Percepción y medida en la reputación empresarial. Economía Industrial, Vol. 3, No 57, pp 117-129.

Solomon, Sandra; Reckers, Philip y Lowe, Jordan (2005). Impact of management image and non-audit services fees on investors' perceptions of earnings quality. Advances in Accounting, Vol. 21, pp 199-216.

Thompson, Allan (1996). Compliance with Agreements in Cross-Cultural Transactions: Some Analytical Issues. Journal of International Business Studies, Second Quarter, pp 375-390.

Weigelt, Keith y Camerer, Colin (1988). Reputation and Corporate Strategy: A Review of Recent Theory and Applications. Strategic Management Journal, Vol. 9, No 5, pp 443-454. 